BNL-114406-2017-CP

\title{
Lost Muon Study for the Muon G-2 Experiment at Fermiab*
}

\author{
S. Ganguly, J. D. Crnkovic \\ Presented at the 8th International Particle Accelerator Conference \\ Bella Conference Center in Copenhagen, Denmark \\ 14-19 May 2017
}

May 2017

Physics Department

Brookhaven National Laboratory

\author{
U.S. Department of Energy \\ USDOE Office of Science (SC), \\ High Energy Physics (HEP) (SC-25)
}




\section{DISCLAIMER}

This report was prepared as an account of work sponsored by an agency of the United States Government. Neither the United States Government nor any agency thereof, nor any of their employees, nor any of their contractors, subcontractors, or their employees, makes any warranty, express or implied, or assumes any legal liability or responsibility for the accuracy, completeness, or any third party's use or the results of such use of any information, apparatus, product, or process disclosed, or represents that its use would not infringe privately owned rights. Reference herein to any specific commercial product, process, or service by trade name, trademark, manufacturer, or otherwise, does not necessarily constitute or imply its endorsement, recommendation, or favoring by the United States Government or any agency thereof or its contractors or subcontractors. The views and opinions of authors expressed herein do not necessarily state or reflect those of the United States Government or any agency thereof. 


\title{
LOST MUON STUDY FOR THE MUON G-2 EXPERIMENT AT FERMILAB *
}

\author{
Sudeshna Ganguly ${ }^{\dagger}$, University Of Illinois At Urbana-Champaign, Urbana, United States of America \\ J. D. Crnkovic, W. M. Morse ${ }^{1}$, Brookhaven National Laboratory, Upton, United States of America \\ D. Stratakis ${ }^{1}$, Fermi National Accelerator Laboratory, Batavia, United States of America \\ on behalf of the Muon g-2 Collaboration
}

\section{Abstract}

The Fermilab Muon $g-2$ Experiment has a goal of measuring the muon anomalous magnetic moment to a precision of $140 \mathrm{ppb}$ - a fourfold improvement over the $540 \mathrm{ppb}$ precision obtained by the BNL Muon $g-2$ Experiment. Some muons in the storage ring will interact with material and undergo bremsstrahlung, emitting radiation and loosing energy. These so called "lost muons" will curl in towards the center of the ring and be lost, but some of them will be detected by the calorimeters. A systematic error will arise if the lost muons have a different average spin phase than the stored muons. Algorithms are being developed to estimate the relative number of lost muons, so as to optimize the stored muon beam. This study presents initial testing of algorithms that can be used to estimate the lost muons by using either double or triple detection coincidences in the calorimeters.

\section{INTRODUCTION}

The muon gyromagnetic $(g)$ ratio is a proportionality constant that relates the muon magnetic moment to the muon spin. Dirac Theory predicts that $g=2$, but a full Quantum Field Theory treatment predicts that $g \neq 2$ for spin- $1 / 2$ point particles like the muon. The (E821) final measurement [1] of the anomalous magnetic moment is

$$
a \equiv \frac{g-2}{2}=116592091(54)(33) \times 10^{-11},
$$

where the first error is statistical and the second error is systematic. The E821 measurement hints at the possibility of new physics with a more than $3 \sigma$ difference $[1,4]$ from the Standard Model prediction.

The anomalous magnetic moment is determined from the anomalous precession frequency $\left(\omega_{a}\right)$ and the average magnetic field $(\vec{B})$ seen by the muons. The anomalous precession frequency for a muon with a momentum perpendicular to the storage ring $\vec{B}$ and electric field $(\vec{E})$ is given by

$$
\vec{\omega}_{a}=-\frac{q}{m}\left[a \vec{B}-\left(a-\frac{1}{\gamma^{2}-1}\right) \frac{\vec{\beta} \times \vec{E}}{c}\right],
$$

where $q$ is the muon charge, $m$ is the muon mass, $\gamma$ is the muon Lorentz factor, $c$ is the speed of light, and $\vec{\beta}$ is the muon

\footnotetext{
* We thank the Fermilab staff and the technical staffs of the participating institutions for their important contributions to this effort. This work was supported by the U.S. Department of Energy, grant DE-SC0015640.

† sgang28@illinois.edu
}

velocity divided by $c$. E821 and the Fermilab Muon g-2 Experiment (E989) [5] run at the so called "magic momentum" $\left(P_{m}=3.094 \mathrm{GeV} / c\right)$ that cancels the $\vec{E}$ contribution to $\omega_{a}$ in Eq. 2:

$$
\vec{\omega}_{a}=-a \frac{q \vec{B}}{m} .
$$

Fits to the decay positron time spectra are used to extract $\omega_{a}$. The 5-parameter fit function [2,3], without the inclusion of systematic effects, that describes g-2 data is:

$$
N(t)=\left(N_{0} / \tau\right) * e^{-t / \tau}\left[1-A \cos \left(\omega_{a} t+\phi\right)\right]
$$

A systematic effect will cause a shift in the $\omega_{a}$ fit value if the effect varies over the time measurement window. The probability for a muon to be lost is not constant in time, as the muons at the outer boundary of the phase space are lost preferentially at early times. The lost muons are expected to have a different average spin polarization than the stored muons due to muons being created at different points in the muon production beamline, leading to a shift in $\omega_{a}$. The relative number of lost muons needs to be minimized and quantified. The E989 goal is to keep the relative muon losses at or below $10^{-4}$ per muon lab frame lifetime $(\tau)$.

Monte Carlo (MC) simulation studies of the E989 muon storage ring can be used to estimate the lost muons. These studies can then be used to optimize the storage of the muon beam by estimating the operating parameters for the inflector magnet, kicker, surface coils, and electrostatic quadrupole system. The inflector magnet provides an almost field free region to transport the beam into the storage region while minimizing perturbations to the storage ring magnetic field. The muons injected into the storage ring are off the central orbit, and the kicker uses a magnetic field to "kick" them into the storage ring acceptance. The surface coils are used to adjust the storage ring magnetic field during operations. The electrostatic quadrupole system uses electric fields to provide weak vertical focusing.

\section{DETECTION OF LOST MUONS}

Muons are lost when they hit the collimators or other materials after injection, curving inward and eventually being lost from the ring. Electrostatic quadrupole scraping [3] removes most of these muons at early times, but a small remaining fraction of the lost muons exist at late times due to the local perturbations in the storage ring fields. The muon beam betatron oscillations also contribute to the muon losses. 


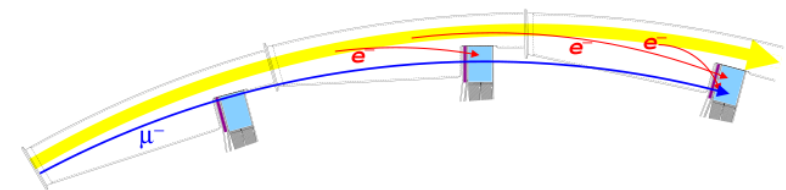

Figure 1: Diagram [2] of a lost muon detected by finding three-fold coincidences among three consecutive calorimeters

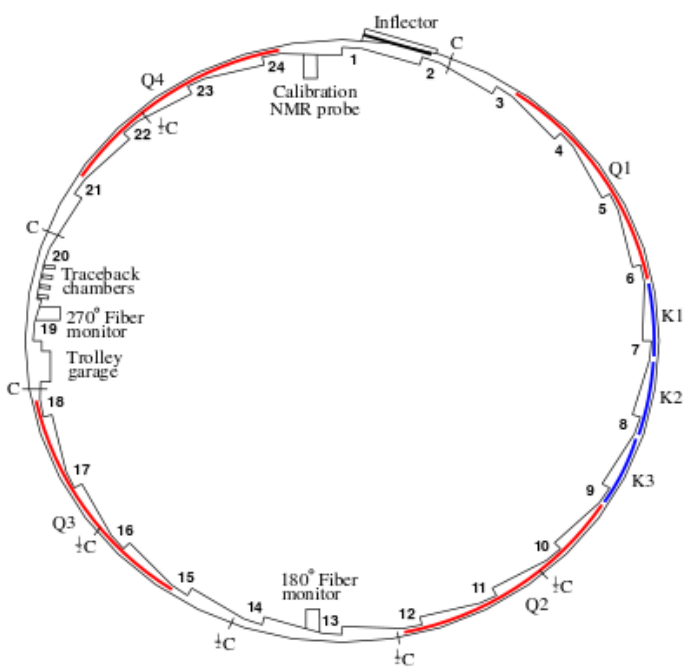

Figure 2: Diagram of the muon g-2 storage ring, The numbers $1-24$ correspond to the calorimeters, $Q_{1}-Q_{4}$ correspond to the electrostatic quadrupoles, $K_{1}-K_{3}$ correspond to the kicker

E989 uses calorimeters [6,7] based on $6 \times 9$ segmented arrays of $\mathrm{PbF}_{2}$ Cherenkov crystals that are placed every $15^{\circ}$ around the storage ring. Two or three detection coincidences in the calorimeter are used to select muon candidates. A muon candidate is a MIP (a minimum ionizing particle) that deposits around $250 \mathrm{MeV}$ in a calorimeter crystal. Typically a muon deposits energy in just one crystal as opposed to decay positrons that deposit energy in a cluster of crystals. Muons can be identified by the $6.25 \pm 0.5$ ns time-of-flight between adjacent calorimeters.

\section{LOST MUON SELECTION CUTS}

A MC simulation of the muon beam starting at the inflector exit has been used to study muon loss. A double coincidence algorithm is used to look at the energy response in two consecutive calorimeters given the signal time window $(\Delta t)$ around the muon time-of-flight between the calorimeters. The double coincidence algorithm uses a $\Delta t$ of $1 \mathrm{~ns}$ between two adjacent calorimeters while the triple coincidence algorithm uses a $\Delta t$ of $1.6 \mathrm{~ns}$ centered on $12 \mathrm{~ns}$ which is the time-of-flight between the first and the third calorimeters. An example $\Delta t$ distribution between two adjacent calorimeters is shown in Fig. 3.

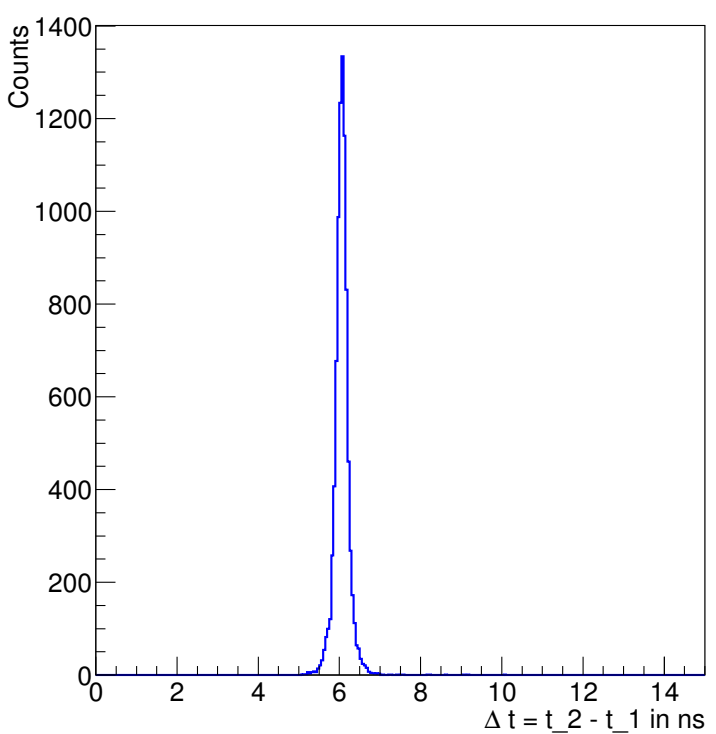

Figure 3: $\Delta t$ between two adjacent calorimeters

A typical simulated $E_{d e p}$ spectrum for coincidence events in two neighboring calorimeters is shown in Fig. 4. Because the muons are MIP-like particles, they produce a Landaulike energy distribution. The low energy tail shown in Fig. 4 is produced by some glancing muons that deposit a very small amount of energy. A selection of $100 \mathrm{MeV}<E_{d e p}$ $<500 \mathrm{MeV}$ will remove the low energy glancing muons as well as the low energy decay positrons that fake MIPs.

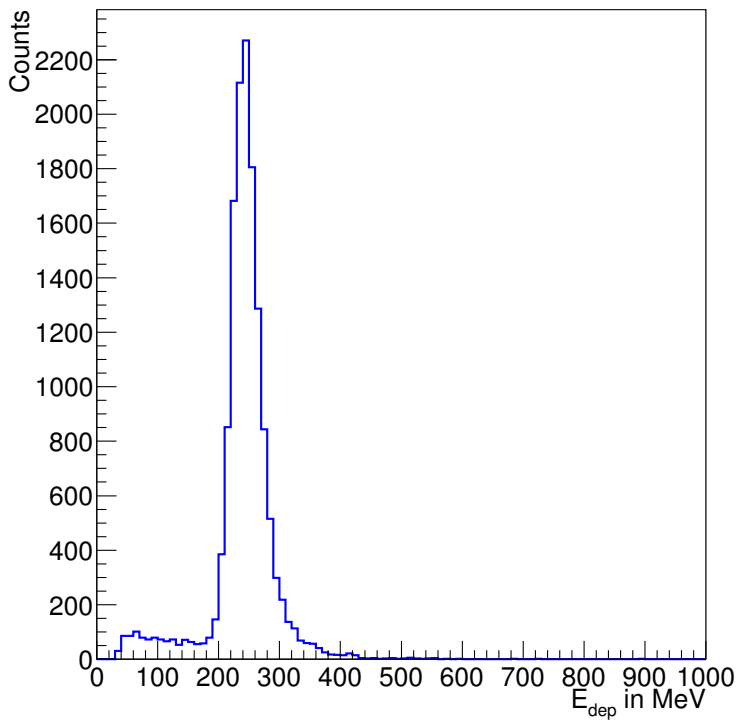

Figure 4: Total energy deposited by a $3 \mathrm{GeV}$ muon detected by the first calorimeter in a double coincidence

A muon mostly deposits all of it's energy in one crystal, but around $30 \%$ of the time energy is shared between neigh- 
boring crystals. A clustering algorithm has been developed by calculating energy weighted position and time (as given by Eq. 4) of the crystal hits in a calorimeter:

$$
\begin{aligned}
X & =\left(\sum E_{x t a l} * X_{x t a l}\right) / \sum E_{x t a l} \\
Y & =\left(\sum E_{x t a l} * Y_{x t a l}\right) / \sum E_{x t a l} \\
t & =\left(\sum E_{x t a l} * t_{x t a l}\right) / \sum E_{x t a l}
\end{aligned}
$$

Figs. 5 and 6 show the energy weighted x vs. energy weighted $y$ for the first and second calorimeter respectively. A muon typically passes through the 9th columns (closest to the storage ring) of the first calorimeter as shown in Fig. 5, then it curls radially inward passing through the central columns of the second calorimeter as shown in Fig. 6

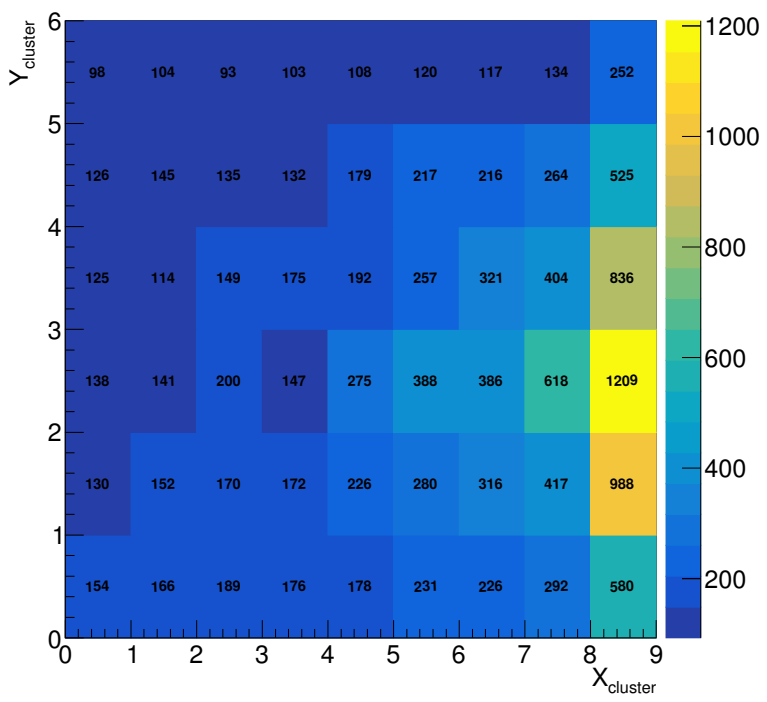

Figure 5: Energy weighted x vs. $y$ on the first calorimeter in a double coincidence

The muon loss signal is expected to be embedded in a much higher background of decay positrons. The decay positrons contribute to two types of backgrounds: uncorrelated two $e^{+}$backgrounds (random coincidences) and correlated one $e^{+}$background. The random coincidences happen when an independent decay positron or second lost muon are randomly detected in the $\Delta t$. The $\Delta t$ contains both correlated and random background coincidence events, while the $\Delta t$ side-bands contain only the random coincidences.

A cut-based analysis has been performed to reduce the correlated in-time-window backgrounds. Three cuts are used in this study: (1) $E_{\text {frac }}=E_{\text {max }} / E_{\text {total }}$, (2) spatial information between the cluster centers in two consecutive calorimeters $X_{2}-X_{1}$, (3) number of crystal hits in a cluster. Cut (1) uses $E_{\text {max }}$ which is the maximum energy in a cluster and $E_{\text {total }}$ which is the total energy in that cluster. $E_{\text {frac }}$ is close to 1 for muons. Cut (2) is used to remove positrons that

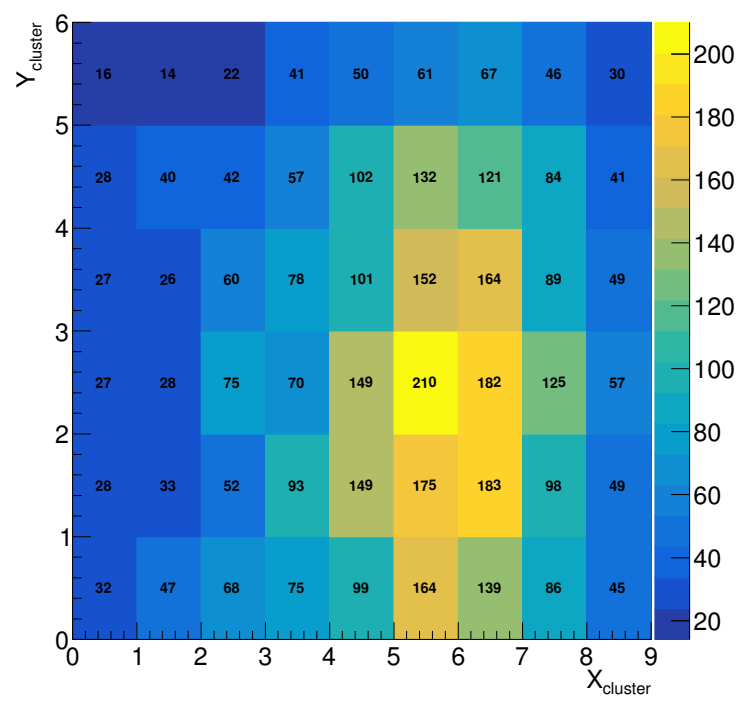

Figure 6: Energy weighted x vs. y on the second calorimeter in a double coincidence

are side entering into the adjacent calorimeter, and therefore predominately hit the crystals closest to the storage volume. Cut (3) selects muons as they produce fewer crystal hits in a cluster compared to the background.

\section{CONCLUSION}

Lost muons systematic effects will lead to a shift in the measured value of $\omega_{a}$. MC simulation of the muon beam and storage ring can be used to estimate the relative number of lost muons, and to optimize the storage ring operating parameters. Algorithms are being developed that use either double or triple calorimeter coincidences to detect the lost muons. These algorithms take advantage of highly segmented calorimeters to separate the muon signal from the much larger decay positron backgrounds.

\section{REFERENCES}

[1] C. Patrignani et al. (Particle Data Group), Chin. Phys. C, 40, 100001 (2016)

[2] C. Polly, A MEASUREMENT OF THE ANOMALOUS MAGNETIC MOMENT OF THE NEGATIVE MUON TO 0.7 PPM, Ph.D. thesis, Phys. Dept., The Graduate College of the University of Illinois at Urbana-Champaign, Urbana, Illinois, United States of America, 2005. [Thesis]

[3] G. W. Bennett et al. [Muon g-2 Collaboration], Phys. Rev. D 73, 072003 (2006) doi:10.1103/PhysRevD.73.072003 [hepex/0602035].

[4] T. Blum, A. Denig, I. Logashenko, E. de Rafael, B. Lee Roberts, T. Teubner and G. Venanzoni, arXiv:1311.2198 [hepph].

[5] J. Grange et al. [Muon g-2 Collaboration], arXiv:1501.06858 [physics.ins-det]. 
[6] J. Kaspar et al., JINST 12, no. 01, P01009 (2017) doi:10.1088/1748-0221/12/01/P01009 [arXiv:1611.03180 [physics.ins-det]].
[7] A. T. Fienberg et al., Nucl. Instrum. Meth. A 783, 12 (2015) doi:10.1016/j.nima.2015.02.028 [arXiv:1412.5525 [physics.ins-det]]. 